\title{
The Relationship Between Job Stress, Job Satisfaction, and the Symptom Checklist-90-Revision (SCL-90-R) in Marine Officers on Board
}

\author{
Jae Hee Kim ${ }^{1}$, Soong-nang Jang² \\ 'Graduate School, Chung-Ang University, Seoul;' ${ }^{2}$ Red Cross College of Nursing, Chung-Ang University, Seoul, Korea
}

Objectives: This study was conducted to investigate the relationships among job stress, job satisfaction, and mental health in marine officers.

Methods: The researchers gathered data on marine officers working at a harbor in Chungcheong Province, South Korea, using a selfreported questionnaire. Mental health was measured by the Symptom Checklist-90-Revision (SCL-90-R), and general characteristics including socioeconomic factors, job stress, and job satisfaction were measured by structured questionnaires. Multiple regression analysis was performed to investigate the relationships among job stress, job satisfaction, and mental health status according to the symptom dimensions of the SCL-90-R.

Results: Among the marine officers, obsessive-compulsive behavior, depression, and somatization were the most problematic symptoms. Those who reported poor health, low job satisfaction, and high job stress had a higher prevalence of psychoticism, somatization, depression, anxiety, and phobic anxiety.

Conclusions: An occupational health system should be introduced that would regularly check the mental health of marine officers in charge of ships and sailors, in order to help reduce their stress levels, enhance their job satisfaction, and thereby improve their mental health.

Key words: Mental health, Stress, Job satisfaction, Ships

\section{INTRODUCTION}

As the stressors that people are exposed to in the workplace can inflict mental pain and increase the risk of developing physical health problems, mental health is an issue that requires preventive interventions. Many studies have suggested

Received: May 14, 2016 Accepted: September 12, 2016

Corresponding author: Soong-nang Jang, PhD

84 Heukseok-ro, Dongjak-gu, Seoul 06974, Korea

Tel: +82-2-820-5806, Fax: +82-2-824-7961

E-mail: sjang@cau.ac.kr

This is an Open Access article distributed under the terms of the Creative Commons Attribution Non-Commercial License (http://creativecommons.org/licenses/by$\mathrm{nc} / 4.0 / /$ which permits unrestricted non-commercial use, distribution, and reproduction in any medium, provided the original work is properly cited. that reduced job satisfaction caused by job stress can lead to increases in employee turnover and absenteeism, and to decreases in productivity and job performance [1].

A marine officer is a person in command of a commercial vessel and the crew. Seafaring is a less preferred job, as seafarers must work under poorer conditions than those in inland occupations, and are also isolated from their homes and society for long periods of time [2]. Marine officers are exposed to highpitched noises, cold spells, heat waves, high temperatures, and unstable conditions with regard to moisture. Since long working hours and frequent work shifts can cause many physiological changes, it is difficult for them to maintain physiological homeostasis $[3,4]$. Such stressful working conditions cause fatigue in sailors and aggravate their stress levels, having a negative 
impact on their health and serving as the cause of manmade mistakes (including safety and maritime accidents) $[5,6]$.

Sailors must leave behind their families and society, maintain limited human relationships in an isolated space, suppress their basic needs, and endure a monotonous life. Clashes, as a result of the cultural diversity of the crew, are inevitable, adding more stress and making it more difficult for individuals to maintain their mental health $[3,4]$. With increased stress, sailors tend to resort to passive measures to cope, including selfisolation or anger expression. If they are exposed to severe stress, their ego function deteriorates, or they may feel anger, inertia, and embarrassment likewise, if they cannot relieve their job-related stress, it can lead to accidents. Due to the unique working environment that isolates them from society, and the fact that the negative influences of stress can be amplified (leading to maritime accidents), more time is required for sailors to recover from stress than for inland workers [7]. Previous studies have suggested that negligence, low safety awareness, job stress, and climate conditions are the major causes of maritime accidents, emphasizing the need to address job-related stress [8].

In a study of the health and stress levels of marine officers, it was found that the risk of developing diseases or health disorders increased by approximately $50 \%$ upon boarding a ship, and that the main causes of this increase were relationships with spouses or partners, lifestyle changes, sleep disorders and dietary problems, stock investment losses and debts, unemployment, dismissals, and conflict with supervisors $[9,10]$. Previous studies on the relationship between job stress and job satisfaction in seafarers have reported that a higher level of job stress led to a decrease in job satisfaction, as well as an increase in turnover intention [4]. However, no studies have been conducted on the relationship between job stress and job satisfaction in Korean seafarers aboard merchant ships. In a study of seafarers who were admitted to the psychiatric department, it was found that the experimental groups who had experience on board deep-sea fishing vessels, were less educated, and who had less than five years of experience on board ships, showed a higher incidence of psychoticism than neurosis. Additionally, the prevalence of somatoform disorder, post-traumatic stress disorder, and other symptomatic mental disorders was higher among seafarers. The results of psychological tests demonstrated that sailors tended to have a narrow mindset, more of a perceived responsibility to support their families, higher levels of disobedience against authority, and more paranoia [11].

The purpose of this study was to identify correlations among job stress, job satisfaction, and the mental health of marine officers on board merchant vessels. The Symptom Checklist90-Revision (SCL-90-R) was employed to examine the potential mental disorders from which marine officers aboard merchant vessels may suffer, and to analyze whether the severity of psychiatric symptoms was influenced by job stress and job satisfaction. The results of this study are expected to be utilized as baseline data to determine measures to improve the mental health of workers engaged in special occupations (including marine officers aboard merchant vessels), and to highlight the necessity for intervention measures and social support to reduce their job stress and improve job satisfaction.

\section{METHODS}

\section{Study Participants}

This study was conducted among Korean marine officers aboard a vessel anchored at Port D. For the data collection, we requested the cooperation of the marine officers aboard the vessel. After explaining the purpose of the study to the officers [12], convenience sampling was carried out on 160 marine officers who consented to participate. The data collection started on June 19, and lasted until September. A total of 149 surveys were used for the final analysis, after excluding eight surveys with answers missing in the major measurement items and three responses from people who were not marine officers. We analyzed the sample size using the $\mathrm{G}^{*}$ Power 3.1.2 program [13] by setting a significance level of 0.05 , a statistical power of $80 \%$, an effect size of 0.15 , and 15 independent variables required for the multiple regression analysis. It was determined that a sample size of 139 individuals was adequate, meaning that our sample satisfied the conditions for the recommended sample size.

\section{Ethical Considerations}

To protect the rights of the participants, this study was conducted after obtaining approval (no. 1041078-201406-HR097-01) from the bioethics committee of the researchers' organization. The researchers explained the purpose of the study and the anonymity and confidentiality agreements to the study subjects, and carried out a questionnaire survey among only those marine officers who consented to participate in the study. 


\section{Measurements}

\section{Marine officers}

Among seafarers, marine officers are those who have a certificate of competency (technology and skill) regarding the operation of ships (issued by the Minister of Oceans and Fisheries) or who have the corresponding licenses (Ship Officer's Act, Article 4) [14]. This study targeted those who held certificates as deck and engineering officers, from first class to sixth class, who are classified according to the scope of their jobs and responsibility.

\section{Job stress}

This study used a modified survey customized for seafarers and based on a simplified tool for the evaluation of job stress in Korean workers [15], with cultural and linguistic variables added. The questionnaire consisted of 31 items in eight subcategories, including job requirements, job autonomy, interpersonal conflicts, job instability, organizational system, inadequate compensation, corporate culture, and language and cultural factors. The negative question items were calculated by reverse conversion. In this study, the consistency among the question items was measured to have a Cronbach's alpha value of 0.856 .

\section{Job satisfaction}

Job satisfaction is a combination of psychological, physical, and environmental factors that allows individuals to feel satisfied with their jobs [16]. This study used a modified evaluation tool [15] customized for seafarers based on the Job Descriptive Index [17]. Each question item was answered using a scale of 0-4; a higher score indicated a higher level of job satisfaction. The questionnaire consisted of nine question items in five subcategories: job satisfaction, the job itself, relationships with supervisors and colleagues, compensation and welfare, and organizational attachment. The negative question items were calculated using reverse conversion. In this study, the consistency among the question items was measured to have a Cronbach's alpha value of 0.68 .

\section{Symptom Checklist-90-Revision}

This study used a multi-dimensional self-reported symptom inventory test that is a standardization of the Global Severity Index (GSI) [18] to be suitable for Koreans. This test consists of 90 question items in nine subcategories, including somatization, obsessive-compulsive behavior, interpersonal sensitivity, depression, anxiety, hostility, phobic anxiety, paranoid ideation, and psychoticism. The test respondents were instructed to select the corresponding scores, depending on the prevalence of symptoms for the last seven days including the day of the test, using a Likert scale of 0 ('never') to 4 ('very often'). The mean reliability of each factor was estimated to have a Cronbach's alpha value of 0.97 . The study used three major global indices: the GSI the Positive Symptom Total (PST), and the Positive Symptom Distress Index (PSDI). The GSI, which is the average score of the 90 items, indicates the current level or depth of a given disorder. The PST is the number of items scored above zero. Lastly, the PSDI, which is calculated as the total sum of the scores of the items scored above zero divided by the PST, indicates the pure intensity of the disorder. The PSDI is also indicative of the response style of the respondents [19].

\section{Data Analysis}

The collected data was analyzed using SPSS version 12.0 (SPSS Inc., Chicago, IL, USA), as follows. The frequency and percentage were calculated to assess the general characteristics of the survey respondents. The mean values and standard deviations of the SCL-90-R, job stress, and job satisfaction were calculated, and the $t$-test and analysis of variance (according to the general and occupational characteristics) were performed. Multiple regression was carried out to assess the influence of each of the 10 psychological diagnosis sub-items (somatization, obsessive-compulsive behavior, interpersonal sensitivity, depression, anxiety, hostility, phobic anxiety, paranoid ideation, psychoticism, and other symptoms) on job stress and job satisfaction. Finally, a multiple regression analysis was conducted to analyze the influence of these items on the GSI, PST, and PSDI, which are the three major global indices included in the SCL-90-R on job stress and job satisfaction, adjusting for self-rated health, body mass index (BMI), job stress, job satisfaction, employment type, compensation, total working period, ship type, and job position.

\section{RESULTS}

\section{Job Stress, Job Satisfaction, and the Symptom Checklist-90-Revision Global Indices, Depending on General Characteristics}

The marine officers who participated in the survey consisted of 149 males, among whom approximately half were in their 20 s and 30s. In terms of the vessel types they were currently 
aboard, oil tankers (including chemical carriers) comprised $74.5 \%$, followed by non-oil tankers (25.5\%). In terms of employment period, subjects were classified as having worked for less than one year, having worked for more than one year but less than six years, having worked for more than six years but less than 15 years, and having worked for more than 15 years, with proportions ranging from $23.5 \%$ to $26.8 \%$.

Among the three global indices of the SCL-90-R, the GDI of the marine officers showed significant differences in terms of age $(F=3.41, p<0.05)$, marital status $(t=-3.09, p<0.01)$ and $\mathrm{BMI}(\mathrm{F}=3.21, p<0.05)$. According to the results of the post-hoc analysis, marine officers who were in their 20s or 30s, who were unmarried, and who were underweight showed relatively higher levels of PSDI. The severity of job stress varied depending on job position $(\mathrm{F}=3.63, p<0.001)$ and employment type $(t=-2.97, p<0.01)$. First mates had the most severe job stress score, followed by second mates, first engineers, and third mates. The levels of job satisfaction varied among age groups $(F=4.40, p<0.05)$, with those 60 or more years old showing higher levels of job satisfaction than those in their 20 s or 30 s (Table 1).

\section{Sub-items of the Symptom Checklist-90-Revi- sion, Job Stress, and Job Satisfaction}

The SCL-90-R can be divided into 10 sub-items. Among the 10 sub-items, the most common psychological symptom was found to be obsessive-compulsive behavior, followed by depression, anxiety, interpersonal sensitivity, and somatization. Among the psychiatric symptom sub-items, psychoticism was measured to be higher than somatization. Among the job stress sub-items, job requirements had the highest score, followed by job autonomy, job instability, and organizational systems. Therefore, job stress was closely linked with job responsibility and organization systems. Among the sub-items of job satisfaction, job relations had the highest score, followed by job satisfaction and the job itself (Table 2).

\section{Factors Associated With Symptom Checklist- 90-Revision Symptom Dimensions}

In order to identify the factors associated with the SCL-90-R symptom dimensions, regression analysis was conducted on each of the 10 sub-items of the SCL-90-R, and the results are as follows. Depression, psychoticism, somatization, and phobic anxiety were found to have a statistically significant correlation with self-rated health. Likewise, low levels of self-rated health had a negative influence on psychological health (as indicated by measures such as depression, psychoticism, and somatization). It was observed that a higher level of job stress had an influence on obsessive-compulsive behavior, depression, and additional items. Unmarried respondents reported higher levels of interpersonal sensitivity, obsessive-compulsive behavior, paranoid ideation, and psychoticism than their married counterparts (Table 3 ).

\section{Factors Influencing the Symptom Checklist- 90-Revision Global Indices}

The factors that influenced the three global indices of the SCL-90-R are presented in Table 4. It was found that the underweight and overweight BMI categories, lower pay, longer total working periods, lower job positions, and temporary employment influenced the GSI and PST global indices. Among the global indices, when all variables were controlled for, lower self-rated health, higher job stress, and the marital status (unmarried) of the respondents significantly influenced the GSI. Self-rated health and job stress were observed to influence the PST.

The factors found to have meaningful associations were different when the general characteristics were modified and when the influence of all the items on the three global indices was measured. However, factors that influenced all three global indices of the SCL-90-R were observed for almost all items. Marine officers showed significant influences in connection with items on the GSI and PST.

\section{DISCUSSION}

This study showed correlations among psychological health, job stress, and job satisfaction in marine officers. Subjects who had higher total SCL-90-R scores (and whose psychiatric symptoms were more severe) showed lower self-rated health and higher job stress. Obsessive-compulsive behavior, depression, and somatization were the most problematic symptoms, and when respondents thought they were unhealthy, they tended to show higher scores in psychoticism, somatization, depression, anxiety, and phobic anxiety. In terms of the PSDI, the third-ranking positions and the underweight respondents showed higher levels of psychiatric symptoms.

The results demonstrated differences in job stress and job satisfaction, and the SCL-90-R global indices depended on the general characteristics of the study subjects. Compared to the 


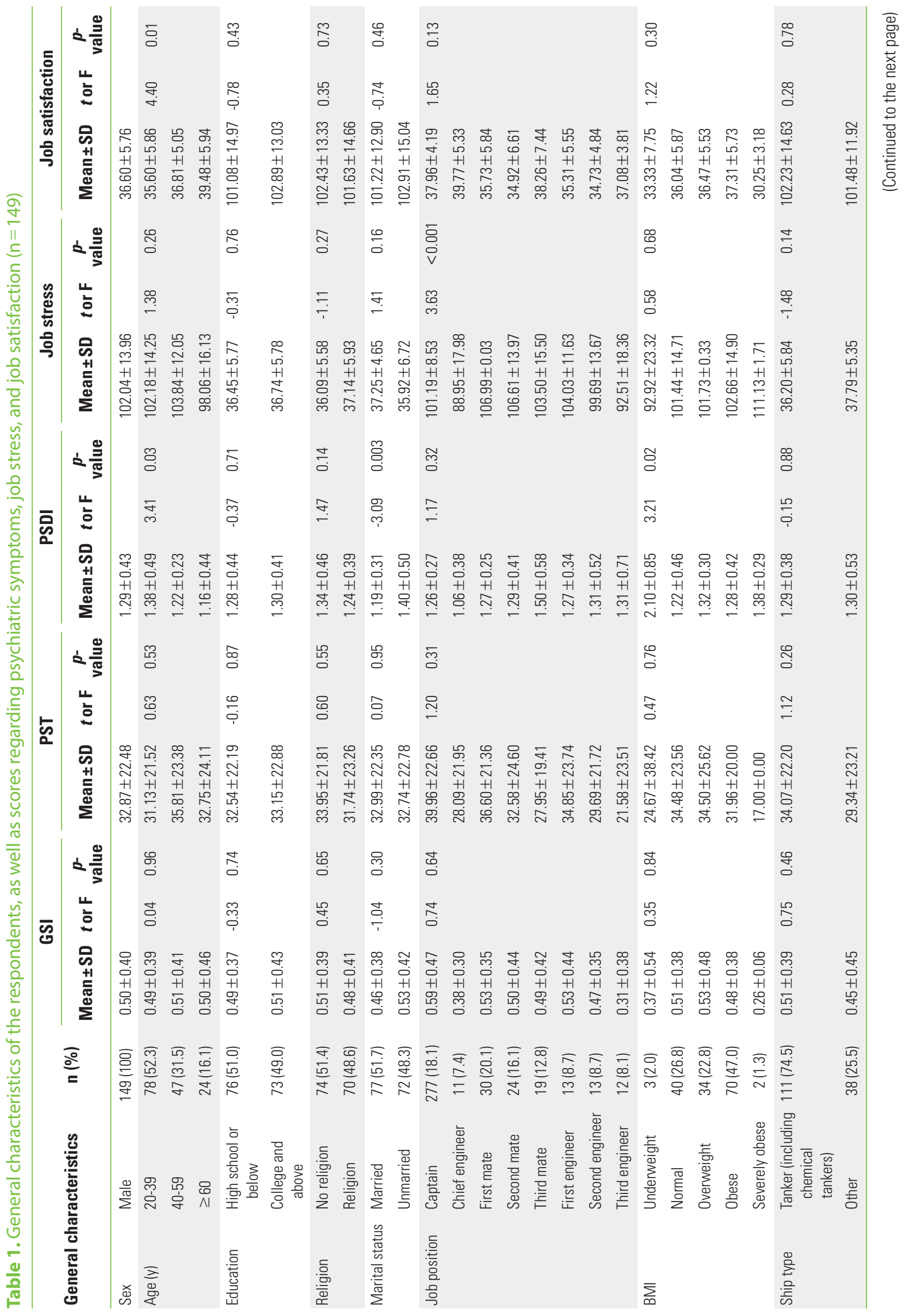




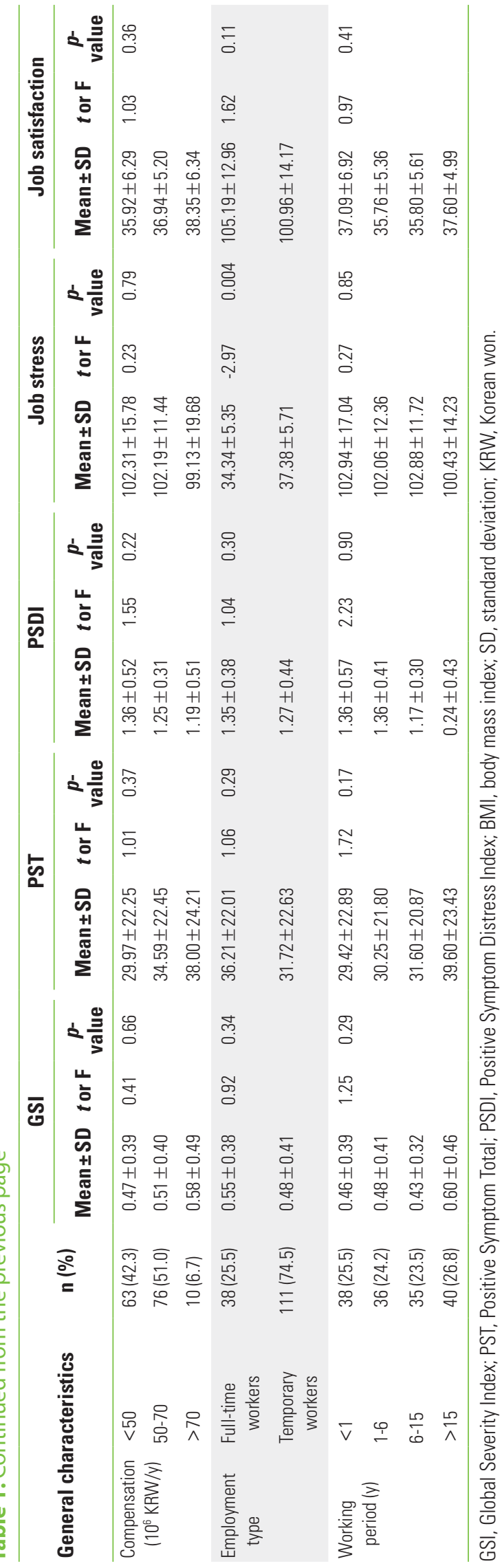

Table 2. SCL-90-R, job stress, and job satisfaction scores $(\mathrm{n}=$ 149)

\begin{tabular}{ll}
\hline Variable & Mean \pm SD \\
\hline SCL-90-R & \\
Somatization & $5.04 \pm 5.36$ \\
Obsessive-compulsive & $7.56 \pm 5.12$ \\
Interpersonal sensitivity & $5.52 \pm 4.47$ \\
Depression & $7.50 \pm 6.71$ \\
Anxiety & $7.05 \pm 7.12$ \\
Hostility & $2.93 \pm 3.83$ \\
Phobic anxiety & $1.56 \pm 2.19$ \\
Paranoid ideation & $3.32 \pm 3.51$ \\
Psychoticism & $3.69 \pm 4.37$ \\
Additional items & $4.13 \pm 3.59$ \\
Job stress & \\
Job requirements & $3.17 \pm 0.75$ \\
Job autonomy & $3.08 \pm 0.39$ \\
Job conflict & $2.55 \pm 0.68$ \\
Job instability & $2.92 \pm 0.74$ \\
Organizational systems & $2.87 \pm 0.64$ \\
Improper compensation & $2.69 \pm 0.62$ \\
Work culture & $2.65 \pm 0.66$ \\
Language and culture & $2.83 \pm 0.51$ \\
Job satisfaction & \\
Job satisfaction & $3.40 \pm 0.55$ \\
Job itself & $3.32 \pm 0.57$ \\
Job relations & $3.64 \pm 0.74$ \\
Compensation & $2.52 \pm 0.91$ \\
Attachment & $2.62 \pm 0.89$ \\
\hline SCl-go-R Sym & \\
\hline & \\
\hline
\end{tabular}

SCL-90-R, Symptom Checklist-90-Revision; SD, standard deviation.

results of a study conducted by Chang et al. [20], which measured the SCL-90-R global indices in ordinary males, the GSI index measured by this study was higher by about one point, while the PSDI was higher by about seven points [20]. This indicates that marine officers presented higher levels of overall disorders, from the perspective of the overall level of pathology, the pure intensity of psychiatric disorders, and the response style, than their ordinary male counterparts. Therefore, marine officers need proper medical care to maintain their mental health. Consistent with the results of a study arguing that being underweight had a negative influence on mental health, this study also observed that being underweight had a negative impact on mental health, which indicates a possible relationship between dietary habits (or nutritional status) and health status. Further studies need to be conducted to confirm this possibility. 


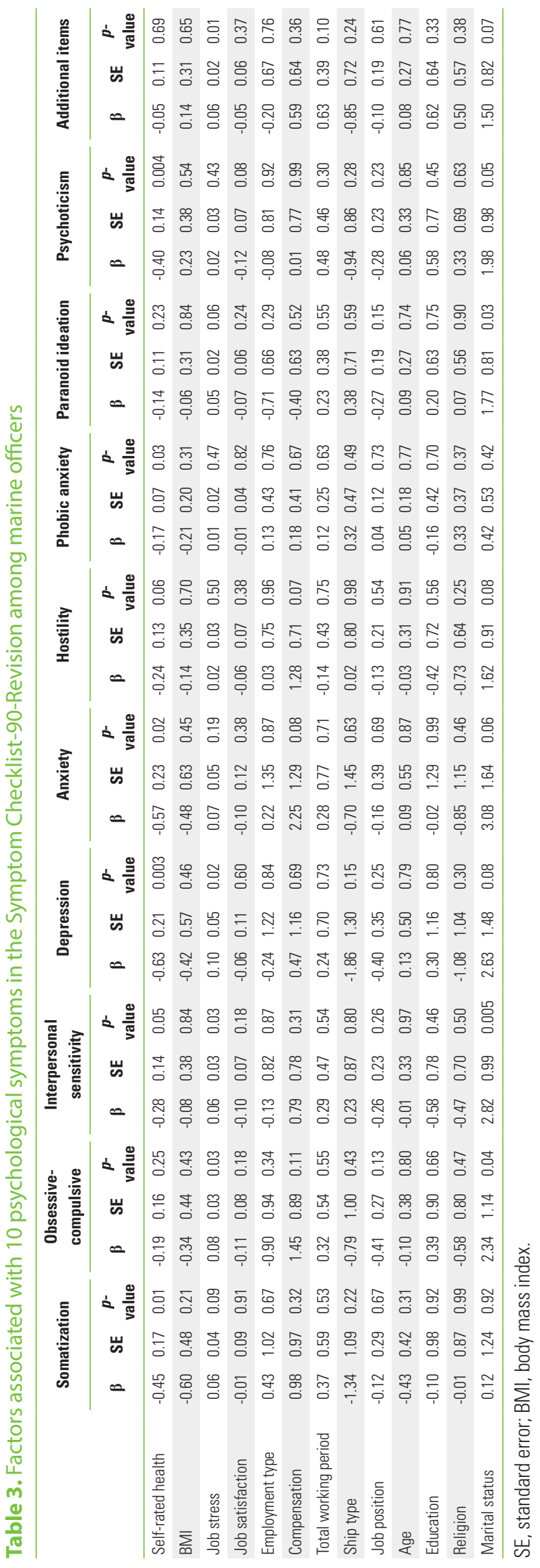

Second, the position of chief engineer had the highest score for job stress, followed by third engineer. Additionally, the engine department displayed a higher level of job stress than the deck department. Third engineers are expected to experience the most severe level of mental and physical stress, resulting from working in the engine room of the ship. Seafarers suffer from various kinds of stress, but among them, noise is the most serious cause of stress in the engine room, and the working and residential environment of the ship is an important factor that influences the mental and physical health of seafarers. Additionally, it has been reported that the frequent arrivals and departures of ships could cause severe mental stress, leading to various health disorders [21]. Therefore, without proper intervention, this pattern can have a negative impact on the mental health of marine officers onboard, leading to maritime or other ship accidents. Previous studies have reported that the job requirements of seafarers are more demanding than those of other occupations, and that the unique corporate culture in Korea (including Korean-style collective culture, irrational communication channels, and unofficial corporate culture) was also a cause of job stress [12].

Third, the factors found to influence job satisfaction were age and employment status. Those in their 20s and 30s had a higher score for job satisfaction than those aged 60 years old and above. These results are consistent with those of another study conducted on seafarers by Kim et al. [22], and this finding can be ascribed to the fact that captains and chief engineers are highly concentrated in the 20 s and 30 s group. Among the job positions, captain had the highest score for job satisfaction, followed by chief engineer [2]. Job satisfaction can influence turnover intentions; an empirical study conducted on turnover among seafarers reported that those with a higher level of satisfaction in their job, social identity, and career vision, as well as those in older age groups, had a lower turnover rate [15]. The chief engineer of a ship, who assumes a management position and has considerable onboard experience, is expected to have a higher job satisfaction score than engineers with lower ranks. In terms of employment type, temporary workers had a higher level of job satisfaction than regular workers, which may be due to the fact that one of the major complaints of regular seafarers is the short breaks (holidays) between disembarkation and the next trip to sea, meaning that irregular seafarers have more autonomy to determine their schedule. Sex differences have yet to be identified. As the previous studies have been conducted mostly among male 
Table 4. Factors associated with the 3 domains of the Symptom Checklist-90-Revision among marine officers

\begin{tabular}{|c|c|c|c|c|c|c|c|c|c|}
\hline & & GSI & & & PST & & & PSDI & \\
\hline & $\beta^{1}$ & SE & $p$-value & $\beta^{1}$ & SE & $p$-value & $\beta^{1}$ & SE & $p$-value \\
\hline Self-rated health & -0.03 & 0.01 & 0.01 & -1.57 & 0.69 & 0.02 & 0.00 & 0.01 & 0.86 \\
\hline BMI & -0.02 & 0.03 & 0.53 & -1.98 & 1.88 & 0.29 & 0.00 & 0.04 & 0.93 \\
\hline Job stress & 0.01 & 0.00 & 0.04 & 0.39 & 0.15 & 0.009 & 0.00 & 0.00 & 0.32 \\
\hline Job satisfaction & -0.01 & 0.01 & 0.28 & -0.46 & 0.35 & 0.19 & 0.00 & 0.01 & 0.60 \\
\hline Employment type & -0.01 & 0.07 & 0.86 & -0.08 & 4.02 & 0.98 & -0.10 & 0.08 & 0.25 \\
\hline Compensation & 0.07 & 0.07 & 0.30 & 3.41 & 3.83 & 0.37 & 0.01 & 0.08 & 0.94 \\
\hline Total working period & 0.03 & 0.04 & 0.47 & 1.70 & 2.31 & 0.46 & 0.01 & 0.05 & 0.91 \\
\hline Ship type & -0.06 & 0.08 & 0.46 & -4.71 & 4.30 & 0.27 & -0.01 & 0.09 & 0.93 \\
\hline Job position & -0.02 & 0.02 & 0.30 & -1.60 & 1.15 & 0.17 & -0.01 & 0.02 & 0.70 \\
\hline Age & 0.00 & 0.03 & 0.10 & 0.29 & 1.64 & 0.86 & -0.06 & 0.03 & 0.10 \\
\hline Education & 0.01 & 0.07 & 0.85 & 0.01 & 3.85 & 0.10 & -0.01 & 0.08 & 0.92 \\
\hline Religion & -0.02 & 0.06 & 0.73 & -1.86 & 3.43 & 0.59 & -0.08 & 0.07 & 0.27 \\
\hline Marital status & 0.18 & 0.09 & 0.04 & 6.47 & 4.89 & 0.19 & 0.13 & 0.10 & 0.19 \\
\hline
\end{tabular}

GSI, Global Severity Index; PST, Positive Symptom Total; PSDI, Positive Symptom Distress Index; BMI, body mass index; SE, standard error.

${ }^{1}$ Beta values were calculated using a regression model adjusting for self-rated health, BMI, employment type, compensation, total working period, ship type, job position, age, education, religion, and marital status.

seafarers, no data supporting possible sex differences exist, although previous studies have claimed that the swift trust formed between seafarers is the most important factor influencing their attitudes and morale and is needed to achieve a high level of job satisfaction and immersion [15]. The second most important factor is the job itself, followed by organizational attachment, compensation, and welfare. The importance of improving the job design and enhancing the social and psychological factors of the job environment in order to increase the level of job satisfaction has been emphasized [23]. Good management and control of the physical environment plays a critical role in improving the job satisfaction and productivity of the organization [24]. Therefore, since job satisfaction is closely linked with job stress, the authors of this study believe that providing a permanent occupational health nurse on a ship will be effective in improving the health and safety of marine officers.

Fourth, the factors influencing self-rated health among the 10 symptom dimensions of the SCL-90-R include psychoticism, which represents a continuum from mild interpersonal alienation to dramatic evidence of psychosis, including isolation, withdrawal, a schizoid lifestyle, hallucinations and thought-broadcasting; somatization, which refers to subjectively perceived distress arising from problems with body systems under the influence of the autonomous nervous system, including headaches and pains; depression, which refers to depressive moods and feelings, according to the clinical diag- nosis criteria, including signs of diminished interest in life, lack of motivation, loss of vital energy, feelings of hopelessness, and suicidal thoughts; anxiety, referring to symptoms associated with nervousness, tension, trembling, feelings of terror and anxiety; and phobic anxiety, describing persistent, irrational and disproportionate fear responses to a specific person, place, object, or situation [18]. People with a sound mind and a healthy body tend to have a high level of self-efficacy. A high level of self-efficacy had a positive influence on the practice of active health improvements in seafarers, and it is believed that a greater perception of being healthy has a positive impact on mental health. Among the 10 symptom dimensions in the SCL-90-R, anxiety had the highest score, followed by depression, phobic anxiety, and somatization; this seems to be closely connected with influential environmental factors, including relationships with spouses or partners and family members, as well as lifestyle changes due to social isolation, which are the most influential cause of stress in seafarers. Psychoticism, phobic anxiety, obsessive-compulsive behavior, and other symptoms also influence job stress and job satisfaction. Comparing the 10 symptom dimensions of the SCL-90-R to the results found among male members of the general population, the male marine officers had higher scores for somatization and hostility than their ordinary male counterparts. The reason the marine officers had a particularly high score in somatization (in the psychiatric symptom test) was that they had frequent complaints about disorders in organs that are sensitive to the 
influence of the autonomous nerve system, in addition to headaches and pains. According to the results of the study, $97.2 \%$ of the 140 respondents said that they have taken painkillers as medication, while $79.9 \%$ of the 115 respondents had taken digestive pills. The issue of providing proper medical care for those seafarers who are left in a blind spot of the medical system still remains unresolved. According to Article 33 of the Ship Officers' Act, "a ship shall be accompanied by a licensed medical officer." Even if marine officers have the appropriate medical licenses, however, it can still be said that no true professional healthcare provider is present. Interventions for mental health at the workplace are known to have a positive influence on the mental stress of employees by helping to reduce job stress, anger, and depression [25].

This study has several limitations, which need to be carefully considered in the interpretation of the study results. The symptom dimensions of the SCL-90-R do not represent clinical diagnoses. Additionally, as this was a cross-sectional correlation study, it is difficult to draw definitive conclusions about the correlations among job stress, job satisfaction, and the SCL-90-R. Since the study was conducted on marine officers whose ships were berthed at a specific port in a certain area, the generalizability of the study results is a concern. All of the evaluation tools were conducted in a self-reported form; as such, they have a limited ability to serve as the basis for objective conclusions regarding correlations among job stress, job satisfaction, and the SCL-90-R.

An occupational health system should be introduced that will regularly monitor the mental health of marine officers in charge of ships and sailors, including evaluations of psychoticism, somatization, depression, anxiety, and phobic anxiety, to help reduce their stress level. Future research is needed to corroborate the correlations among job stress, job satisfaction, and mental health, and to develop health promotion program interventions to improve their job environment.

\section{ACKNOWLEDGEMENTS}

This study was supported by the Chung-Ang University Research Scholarship Grant in 2014.

\section{CONFLICT OF INTEREST}

The authors have no conflicts of interest associated with the material presented in this paper.

\section{ORCID}

Jae Hee Kim http://orcid.org/0000-0003-4484-2114

Soong-nang Jang http://orcid.org/0000-0003-2621-945X

\section{REFERENCES}

1. Gershon RR, Barocas B, Canton AN, Li X, Vlahov D. Mental, physical, and behavioral outcomes associated with perceived work stress in police officers. Crim Justice Behav 2000;36(3): 275-289.

2. Lee HS. A study on the use and satisfaction of the shipping companies' welfare program by the characteristics of crew's demography and working condition on board. J Navig Port Res 2012;36(3):149-155 (Korean).

3. Bokti NL, Talib MA. A preliminary study on occupational stress and job satisfaction among male navy personnel at a naval base in Lumut, Malaysia. J Int Soc Res 2009;2(9):299-307.

4. Kim JH. A study on the improvement method for health management of seafares. J Navig Port Res 2013;37(1):29-34 (Korean).

5. Shcherbina FA, Myznikov IL. Compensation and adaptation in sailors during voyages of different lengths. Hum Physiol 2004; 30(1):105-110.

6. Żychlińska M, Żychliński M, Siermontowski P. Clinical aspect of naval crews combat stress. Pol Hyperb Res 2016;55(2):29-38.

7. Seo YS, Kim JH. A preliminary study on the stress perception and ways to cope with stress for seafarers. J Navig Port Res 2005;29(1):35-42 (Korean).

8. Chauvin C, Lardjane S, Morel G, Clostermann JP, Langard B. Human and organisational factors in maritime accidents: analysis of collisions at sea using the HFACS. Accid Anal Prev 2013;59:26-37.

9. Akerstedt T. Psychosocial stress and impaired sleep. Scand J Work Environ Health 2006;32(6):493-501.

10. Rydstedt LW, Lundh M. An ocean of stress? The relationship between psychosocial workload and mental strain among engine officers in the Swedish merchant fleet. Int Marit Health 2010;62(3):168-175.

11. Lee JH, Kim JH, Kim JG. A study of sailor psychiatric inpatients: focusing on the transition in a general hospital. J Korean Neuropsychiatr Assoc 1997;36(6):1004-1011 (Korean).

12. Lee JS, Kim TH, Shin YJ. The impact of communication and conflict on group cohesiveness and organization effectiveness in ship organization. J Navig Port Res 2012;36(6):489-499 
(Korean).

13. Faul F, Erdfelder E, Lang AG, Buchner A. G*Power 3: a flexible statistical power analysis program for the social, behavioral, and biomedical sciences. Behav Res Methods 2007;39(2):175191.

14. Sampson $H$, Zhao M. Multilingual crews: communication and the operation of ships. World English 2003;22(1):31-43.

15. Kim JM, Lee DH. The determinants of turnover intentions of Korean seafarers. J Navig Port Res 2011;35(3):219-226 (Korean).

16. Judge TA, Thoresen CJ, Bono JE, Patton GK. The job satisfaction-job performance relationship: a qualitative and quantitative review. Psychol Bull 2001;127(3):376-407.

17. Roznowski M. Examination of the measurement properties of the Job Descriptive Index with experimental items. J Appl Psychol 1989;74(5):805-814.

18. Derogatis LR, Cleary PA. Confirmation of the dimensional structure of the SCL-90: a study in construct validation. J Clin Psychol 1977;33(4):981-989.

19. Vaurio R. Symptom Checklist-90-Revised. In: Kreutzer JS, DeLuca J, Caplan B, editors. Encyclopedia of clinical neuropsy- chology. New York: Springer; 2011, p. 2447-2450.

20. Chang JH, Kim D, Jang E, Park JE, Bae H, Han CW, et al. Reliability and validity of the Korean version of the Symptom Checklist-Post-Traumatic Stress Disorder Scale. J Korean Med Sci 2016;31(5):777-782.

21. Carotenuto A, Molino I, Fasanaro AM, Amenta F. Psychological stress in seafarers: a review. Int Marit Health 2012;63(4):188194.

22. Kim HT, Na S, Ha WH. A case study of marine accident investigation and analysis with focus on human error. J Ergon Soc Korea 2011;30(1):137-150 (Korean).

23. Peterson M, Dunnagan T. Analysis of a worksite health promotion program's impact on job satisfaction. J Occup Environ Med 1998;40(11):973-979.

24. Kim SL, Jung HS, Lee BI, Lee JE, Rhee KY. Factors related with job satisfaction in workers: through the application of $\mathrm{NIOSH}$ job stress model. J Korean Community Nurs 2003;14(2):190198 (Korean).

25. Sin HS, Youn DG. The Effect of captain's transformational leadership on job satisfaction of KCG. J Korean Soc Mar Environ Saf 2013;19(1):23-30 (Korean). 